\title{
Avaliação da multiplicação de Listeria monocytogenes e da atividade antimicrobiana do Biomax $D^{\circledR}$ em nata
}

\section{Evaluation of multiplication of Listeria monocytogenes and antimicrobial activity of Biomax $D^{\circledR}$ in cream}

\author{
Ana Paula Biasus ${ }^{1}$, Ana Paula Chaves ${ }^{1}$, Wladimir Padilha da Silva², Cleuzir da Luz e Liziane \\ Schittler ${ }^{*}$
}

\author{
${ }_{1}^{1}$ Departamento de Engenharia de Alimentos, Centro de Educação Superior do Oeste, Universidade do Estado de Santa Catarina - UDESC, CEP 89870-000, Pinhalzinho/SC, Brasil; \\ 2 Departamento de Ciência e Tecnologia Agroindustrial, Universidade Federal de Pelotas - UFPel, CEP:96010-900, Pelotas/RS, Brasil. \\ (*E-mail: lizianeschittler@gmail.com) \\ http://dx.doi.org/10.19084/RCA15084
}

Recebido/received: 2015.07 .15

Aceite/accepted: 2015.11.17

\section{R E S U M O}

Avaliou-se a multiplicação de Listeria monocytogenes em nata, um derivado lácteo com alto teor de gordura, bem como a atividade antilisteria do Biomax $\mathrm{D}^{\circledR}$ neste alimento. A atividade antilisteria do Biomax foi comparada com a da nisina. Foram utilizados seis tratamentos: T1: nata $+10^{2}$ UFC.g-1 de L. monocytogenes; T2: nata $+10^{2} \mathrm{UFC}^{-g^{-1}}$ de L. monocytogenes + 2,5ppm de nisina; T3: nata + $10^{2}$ UFC. ${ }^{-1}$ de L. monocytogenes $+1,5 \%$ de Biomax; T4: nata + de $10^{5}$ UFC.g- ${ }^{-1}$ de L. monocytogenes; T5: nata + de $10^{5}$ UFC.g-1 de L. monocytogenes + 2,5ppm de nisina; T6: nata + de $10^{5} \mathrm{UFC}^{-g^{-1}}$ de L. monocytogenes + $1,5 \%$ de Biomax. A contagem de L. monocytogenes foi realizada em três tempos de armazenamento a $8^{\circ} \mathrm{C}\left(\right.$ zero, $6^{\circ}, 14^{\circ}$ e $20^{\circ}$ dia), com médias variando entre 2 e $10 \log$ UFC. $\mathrm{g}^{-1}$ nos tratamentos sem adição de nisina e Biomax, entretanto, houve eliminação do micro-organismo nos tratamentos adicionados dos antimicrobianos. Verificou-se que L. monocytogenes se desenvolve em nata, podendo atingir elevados níveis de contaminação, mesmo quando apresentava baixas contagens iniciais. Além disso, observou-se que Biomax possui atividade antilisteria semelhante à nisina neste derivado lácteo, apresentando potencial para ser utilizado como antimicrobiano natural para o controle de L. monocytogenes em alimentos gordurosos.

Palavras-chave: antimicrobianos, Biomax $\mathrm{D}^{\circledR}$, nata, nisina.

\begin{abstract}
A B S T R A C T
The proliferation of Listeria monocytogenes in cream, a milk derivative with high fat content, was evaluated as well as the anti-listerial activity of Biomax $\mathrm{D}^{\circledR}$ in this type of food. Antilisterial activity of Biomax was compared with nisin. Six treatments were used: T1: Cream $+10^{2}$ CFU.g ${ }^{-1}$ of L. monocytogenes; T2: Cream $+10^{2} \mathrm{CFU} \cdot \mathrm{g}^{-1}$ L. monocytogenes $+2,5 \mathrm{ppm}$ nisin; T3: Cream + 102 CFU.g-1 L. monocytogenes + 1.5\% Biomax; T4: Cream + 105 CFU.g-1 of L. monocytogenes; T5: Cream + $10^{5}$ CFU.g-1 L. monocytogenes $+2,5$ ppm nisin; T6: Cream $+10^{5}$ CFU.g-1 L. monocytogenes $+1.5 \%$ Biomax. L. monocytogenes counts were performed on three days storage at $8^{\circ} \mathrm{C}$ (zero, 6,14 and $20^{\text {th }}$ day), with averages ranging between 2 and 10 $\log$ CFU.g-1 in treatments without the addition of nisin or Biomax ${ }^{\circledast}$. However, there was elimination of the microorganism when the antimicrobials were added. It was found that L. monocytogenes is able to grow in cream, reaching high levels of contamination, even when it presents low initial counts. In this dairy derivative, Biomax $\mathrm{D}^{\circledR}$ presents antilisterial activity similar to nisin and might be used as a natural antibiotic for the control of L. monocytogenes in fatty foods.
\end{abstract}

Keywords: antimicrobials; Biomax $\mathrm{D}^{\circledR}$, cream, nisin. 


\section{INTRODUÇÃo}

Atualmente, há uma busca por alimentos mais saudáveis, seguros, e com aspecto mais próximo ao natural. Para atender esta demanda do mercado, as indústrias de alimentos têm utilizado a bioconservação, que consiste na substituição de antimicrobianos obtidos através de síntese química, por compostos naturais de origem microbiana ou vegetal, com atividade antimicrobiana comprovada.

Um exemplo é a nisina, um peptídeo antimicrobiano sintetizado por bactérias lácticas da espécie Lactococcus lactis, que tem sido utilizada como conservante pelas indústrias de laticínios, em produtos como queijo e requeijão. Essa bacteriocina possui atividade antagonista contra vários micro-organismos patogênicos, como Clostridium botulinum, Bacillus cereus e Listeria monocytogenes (Jack et al., 1995). Além da nisina, outras substâncias antimicrobianas naturais têm sido descritas, como, por exemplo, o produto a base de extrato de pomelo e ácido ascórbico, denominado comercialmente de Biomax $\mathrm{D}^{\circledR}$.

O Biomax $\mathrm{D}^{\circledR}$ não altera a cor, sabor e odor dos alimentos e tem sido utilizado para o controle de bolores e leveduras na indústria de panificação, entretanto, também apresenta atividade antimicrobiana contra bactérias como C. botulinum, Staphylococcus aureus, Streptococcus hemolyticus, Bacillus stearothermophilus, Bacillus subtilis e L. monocytogenes (Prozin, 2013). Esse produto é comercializado no Brasil, e não necessita registro por apresentar extratos de plantas em sua formulação, conforme preconiza a Resolução no 23 , de 15 de março de 2000, que dispõe sobre Procedimentos básicos para registro e dispensa da obrigatoriedade de registro de produtos pertinentes à área de alimentos (Brasil, 2000).

Listeria monocytogenes é uma bactéria intracelular facultativa que pode causar a listeriose, uma doença que é, principalmente, transmitida por alimentos. Produtos lácteos têm sido identificados como fontes de surtos e casos de listeriose (Cossart, 2011), sendo o leite cru, um veículo importante de contaminação de laticínios por $L$. monocytogenes (Tenenhaus-Aziza et al., 2014). Este micro-organismo apresenta a capacidade de colonizar superfícies em plantas de processamento de alimentos e de formar biofilmes, o que pode contribuir para sua persistência em indústrias de beneficiamento de leite e derivados.

A nata é um produto lácteo, relativamente rico em gordura (38-50\%), que se apresenta como uma emulsão de gordura em água (Brasil, 2012). Apesar de apresentar uma concentração elevada de gordura e ser mantida sob refrigeração, é um alimento pronto para consumo e que pode permitir a multiplicação de bactérias patogênicas psicrotróficas, como L. monocytogenes. Desta forma, a nata pode ser veículo de transmissão de listeriose, entre outras doenças de origem alimentar. Aliado a isso, a composição química da matriz alimentícia pode interferir na atividade do antimicrobiano utilizado, em especial alimentos com alto teor de proteínas ou de gorduras (Busatta et al., 2008).

Devido ao exposto, este trabalho teve como objetivo avaliar a multiplicação de L. monocytogenes em nata, bem como a atividade antimicrobiana de Biomax $\mathrm{D}^{\circledR}$ contra esse patógeno. Além disso, objetivou-se comparar a atividade do Biomax $\mathrm{D}^{\circledR}$ com a nisina, um antimicrobiano natural amplamente utilizado em derivados lácteos.

\section{MATERIAL E MÉTODOS}

Os antimicrobianos nisina e o produto composto por extrato de pomelo e ácido ascórbico, comercializados com os nomes de Globalnissin ${ }^{\circledR}$ e Biomax $\mathrm{D}^{\circledR}$, respectivamente, foram fornecidos gentilmente pelas empresas Globalfood Sistemas, Ingredientes e Tecnologia para Alimentos Ltda e Prozyn Indústria e Comércio Ltda, respectivamente.

A cepa L. monocytogenes Scott A, utilizada neste estudo, pertence à coleção do Laboratório de Microbiologia do curso de Engenharia de Alimentos da UDESC. A cultura, mantida em Tryptic Soy Broth Yeast Extract (TSB-YE/OXOID) adicionado de 1,5\% de ágar-ágar (OXOID), foi repicada em caldo Infusão de Cérebro e Coração (BHI/OXOID) e incubada a $30^{\circ} \mathrm{C}$ por 24 horas. Em seguida, diluiu-se a cultura em solução salina peptonada a 0,1\% (Merck) até a concentração de 0,5 da escala de Mac Farland, correspondente a $10^{8}$ Unidades Formadoras de Colônias por mililitro (UFC $\left.\mathrm{mL}^{-1}\right)$.

Utilizou-se nata de leite bovino, adquirida no comércio, procedente de dois lotes distintos, a qual foi avaliada microbiologicamente pela contagem 
total de micro-organismos aeróbios mesófilos, de acordo com a Instrução Normativa № 62, do Ministério da Agricultura, Pecuária e Abastecimento (Brasil, 2003).

Para avaliar a atividade antimicrobiana de Biomax $\mathrm{D}^{\circledR}$ contra L. monocytogenes em nata, foram utilizados seis tratamentos contendo $200 \mathrm{~g}$ de nata cada: Tratamento 1: nata $+10^{2}$ UFC. $g^{-1}$ de L. monocytogenes; Tratamento 2: nata $+10^{2} \mathrm{UFC}^{-1}$ de L. monocytogenes $+2,5$ ppm de nisina; Tratamento 3: nata + 102 UFC.g-1 de L. monocytogenes $+1,5 \%$ de Biomax $\mathrm{D}^{\circledR}$; Tratamento 4: nata + de $10^{5} \mathrm{UFC}^{-g^{-1}}$ de L. monocytogenes; Tratamento 5: nata + de $10^{5} \mathrm{UFC}^{-g^{-1}}$ de L. monocytogenes + 2,5ppm de nisina; Tratamento 6: nata + de $10^{5} \mathrm{UF}-$ C.g ${ }^{-1}$ de L. monocytogenes $+1,5 \%$ de Biomax D®. Cada tratamento foi homogeneizado em Stomacher (Logen Scientific), e a nata foi fracionada em embalagens plásticas esterilizadas, as quais foram armazenadas a $8^{\circ} \mathrm{C}$. $\mathrm{O}$ experimento foi realizado em duplicata, com natas fabricadas em datas diferentes. A contagem de L. monocytogenes foi realizada logo após o preparo dos tratamentos (tempo zero), no sexto, $14^{\circ}$ e $20^{\circ}$ dia de armazenamento a $8^{\circ} \mathrm{C}$. Para isto, de cada tratamento foram transferidas, assepticamente, $10 \mathrm{~g}$ de amostra para sacos plásticos esterilizados tipo stomacher e adicionou-se $90 \mathrm{~mL}$ de solução salina peptonada a $0,1 \%$. Foram inoculados $0,1 \mathrm{~mL}$ das diluições apropriadas na superfície das placas de Petri contendo ágar Oxford (Oxoid) as quais foram incubadas a $37 \pm 1^{\circ} \mathrm{C}$ por $48 \mathrm{~h}$. As placas que apresentaram colônias pequenas, com aproximadamente 2 $\mathrm{mm}$, acinzentadas, com halos enegrecidos e centro côncavo, foram contadas e o resultado foi expresso em log UFC.g ${ }^{-1}$.
Os dados experimentais referentes às contagens de L. monocytogenes, obtidas em cada tratamento, foram ajustados por funções matemáticas pelo Método de Mínimos Quadrados utilizando o software Excel 2007.

Os resultados das contagens foram submetidos à análise de variância (ANOVA), seguida do teste de Tukey, com nível de 95\% de confiabilidade. Utilizou-se o software livre Assistat 7.7beta.

\section{RESULTADOS E DISCUSSÃO}

No Quadro 1 podem ser visualizadas as contagens de L. monocytogenes em nata submetidas aos tratamentos com e sem os antimicrobianos nisina e Biomax $\mathrm{D}^{\circledR}$ nos tempos zero (logo após o preparo), sexto, $14^{\circ}$ e $20^{\circ}$ dia de armazenamento a $8^{\circ} \mathrm{C}$.

Os Tratamentos 1, 4, 5 e 6 foram ajustados pelas funções polinomiais de grau 2 (dois), pois apresentaram os melhores coeficientes de determinação $\left(R^{2}\right)$, mais próximos de 1 , que estão representadas nas equações 1 a 4 , respectivamente:

$y=0,042 x^{2}+0,2885 x+2,0848 \quad R^{2}=0,9968$

(Eq.1)

$y=0,0131 x^{2}-0,0188 x+5,129 \quad R^{2}=0,9977$

(Eq.2)

$y=0,0131 x^{2}-0,0188 x+5,129 \quad R^{2}=0,9884$

(Eq.3)

Quadro 1 - Médias das contagens $\left(\log _{10}\right.$ UFC.g-1) na nata após os tratamentos com L. monocytogenes e os antimicrobianos durante 20 dias de armazenamento a $8^{\circ} \mathrm{C}$. Tratamento 1: nata $+10^{2} \mathrm{UFC} . \mathrm{g}^{-1} \mathrm{de}$ L. monocytogenes; Tratamento 2: nata + $10^{2}$ UFC.g- ${ }^{-1}$ de L. monocytogenes $+2,5 \mathrm{ppm}$ de nisina; Tratamento 3: nata $+10^{2} \mathrm{UFC}^{-g^{-1}}$ de L. monocytogenes $+1,5 \%$ de Biomax $\mathrm{D}^{\circledR}$; Tratamento 4: nata + de $10^{5} \mathrm{UFC} \mathrm{g}^{-1}$ de $L$. monocytogenes; Tratamento 5: nata + de $10^{5}$ UFC.g.-1 de $L$. monocytogenes + 2,5ppm de nisina; Tratamento 6: nata + de $10^{5} \mathrm{UFC}^{-1}{ }^{-1}$ de L. monocytogenes + 1,5\% de Biomax $\mathrm{D}^{\circledR}$

\begin{tabular}{|c|c|c|c|c|}
\hline \multirow{2}{*}{ Tratamento } & \multicolumn{4}{|c|}{ Tempo (dias) } \\
\hline & $\mathbf{0}$ & 6 & 14 & 20 \\
\hline 1 & $2,00^{\mathrm{aA}} \pm 0,00$ & $4,18^{\mathrm{bA}} \pm 0,57$ & $6,74^{\mathrm{cA}} \pm 0,04$ & $9,63^{\mathrm{dA}} \pm 0,61$ \\
\hline 2 & $1,16^{\mathrm{aB}} \pm 0,15$ & $2,14^{\mathrm{aB}} \pm 0,99$ & $1,00^{\mathrm{aB}} \pm 0,00$ & $1,00^{\mathrm{aB}} \pm 0,00$ \\
\hline 3 & $1,44^{\mathrm{aB}} \pm 0,40$ & $1,77^{\mathrm{aB}} \pm 0,68$ & $1,16^{\mathrm{aB}} \pm 0,15$ & $1,00^{\mathrm{aB}} \pm 0,00$ \\
\hline 4 & $5,08^{\mathrm{aA}} \pm 0,17$ & $5,61^{\mathrm{bA}} \pm 0,22$ & $7,31^{\mathrm{cA}} \pm 0,33$ & $10,04^{\mathrm{dA}} \pm 0,12$ \\
\hline 5 & $4,23^{\mathrm{aA}} \pm 0,76$ & $4,06^{\mathrm{aAB}} \pm 1,05$ & $3,19^{\mathrm{aB}} \pm 1,09$ & $1,00^{\mathrm{bB}} \pm 0,00$ \\
\hline 6 & $4,24^{\mathrm{aA}} \pm 0,02$ & $3,74^{\mathrm{bB}} \pm 0,018$ & $3,08^{\mathrm{abB}} \pm 0,58$ & $1,00^{\mathrm{cB}} \pm 0,00$ \\
\hline
\end{tabular}

ABLetras maiúsculas diferentes nas colunas indicam diferença estatística pelo teste de Tukey $(p<0,05)$ entre os tratamentos. abLetras minúsculas diferentes nas linhas indicam diferença estatística pelo teste Tukey $(p<0,05)$ entre o tempos de armazenamento. Letras iguais não diferem entre si significativamente. 
$y=-0,094 x^{2}+0,0371 x+4,1303 \quad R^{2}=0,9714$

(Eq.4)

Já os Tratamentos 2 e 3, foram ajustados pelas funções polinomiais de grau 3 (três), estando representadas pelas equações 5 e 6 , respectivamente:

$y=0,0016 x^{3}-0,0539 x^{2}+0,4289 x+1,16 \mathrm{R}^{2}=1$

$y=0,0006 x^{3}-0,0223 x^{2}+0,1655 x+1,44 \mathrm{R}^{2}=1$

(Eq.6)

Observa-se que nos Tratamentos 1 e 4, onde foi adicionado à nata somente L. monocytogenes, nas concentrações de $10^{2}$ e $10^{5}$ UFC. ${ }^{-1}$, respectivamente, as médias das contagens no $20^{\circ}$ dia de armazenamento foram de 9,63 log UFC. ${ }^{-1}$ no Tratamento 1 e $10 \log$ UFC.g-1 $^{-1}$ no Tratamento 4, não havendo diferença significativa $(p<0,05)$ entre essas contagens (Quadro 1). Este resultado demonstra que $L$. monocytogenes pode se desenvolver em nata durante o período de armazenamento e chegar a concentrações celulares elevadas, mesmo quando o produto apresenta baixas concentrações iniciais $\left(10^{2}\right.$ UFC. $\left.^{-1}\right)$ do micro-organismo. Resultado semelhante foi relatado por Pimentel-Filho et al. (2014), que inocularam $L$. monocytogenes em queijo Minas Frescal, numa concentração inicial de $10^{3} \mathrm{UFC}^{-g^{-1}} \mathrm{e}$, após 12 dias de armazenamento a $8-10^{\circ} \mathrm{C}$, a concentração desse micro-organismo atingiu $10^{8}$ UFC.g-1.

Quando foram adicionados nisina ou Biomax $\mathrm{D}^{\circledR} \mathrm{à}$ nata (Tratamentos 2, 3, 5 e 6) observou-se que houve uma rápida ação das substâncias antimicrobianas, havendo redução de aproximadamente $1 \log$ UFC. $g^{-1}$ na contagem de L. monocytogenes logo após o preparo dos Tratamentos (tempo zero) em comparação aos Tratamentos 1 e 4, sem adição dos antimicrobianos (Quadro 1). Comportamento similar foi relatado por Bhatti et al. (2004), os quais verificaram uma redução de 1 a $2 \log$ na concentração de L. monocytogenes logo após a adição de 3 ppm de nisina em leite pasteurizado adicionado de $4 \log$ do micro-organismo.

Alguns autores, como Aasen et al. (2003) e Busatta et al. (2008), relatam que o teor de gordura pode influenciar a eficácia dos antimicrobianos. Porém, neste trabalho, verificou-se que a nisina, bem como o Biomax $\mathrm{D}^{\otimes}$, apresentaram atividade antimicrobiana contra L. monocytogenes em nata, que é um derivado lácteo que apresenta uma concentração alta de gordura (38 - 50\%).

Neste estudo utilizou-se a nata que passou pelo processo de homogeneização da gordura. De acordo com Bhatti et al. (2004), a atividade antilisteria da nisina está diretamente relacionada com a forma que se encontra o glóbulo de gordura no leite: utilizando 125 UI.mL ${ }^{-1}$ nisina, o que corresponde a 3 ppm de nisina, contra 4 log de L. monocytogenes em leite in natura com 3,5\% de gordura, o micro-organismo permaneceu viável na concentração de 3 $\log$ UFC. $\mathrm{mL}^{-1}$ no $15^{\circ}$ dia de armazenamento a $5^{\circ} \mathrm{C}$, porém, em leite homogeneizado e pasteurizado, houve eliminação de L. monocytogenes no sexto dia de armazenamento a $5^{\circ} \mathrm{C}$. A atividade antimicrobiana da nisina pode variar de acordo com a absorção na superfície a qual foi incorporada. Isso ocorre pela diferença no tamanho do glóbulo de gordura, tendo em vista que este apresenta tamanho de $3 \mu \mathrm{m}$ no leite in natura e, no leite homogeneizado, apresenta $0,5 \mu \mathrm{m}$.

Avaliando-se o Quadro 1, observa-se que quando se utilizou a interação entre $2,5 \mathrm{ppm}$ de nisina e $L$. monocytogenes na concentração de $10^{2} \mathrm{UFC}^{-1}{ }^{-1}$ (Tratamento 2), houve a redução para $1 \log \mathrm{UFC} \cdot \mathrm{g}^{-1} \mathrm{do}$ micro-organismo no $14^{\circ}$ dia de armazenamento. Já no Tratamento 3, onde foi testado 1,5\% de Biomax $\mathrm{D}^{\circledR} \mathrm{em}$ L. monocytogenes, também na concentração de $10^{2}$ UFC. $\mathrm{g}^{-1}$, a concentração microbiana reduziu para 1,16 log UFC.g-1 ${ }^{-1}$ no mesmo período de tempo. No entanto, comparando-se as médias, verifica-se que não houve diferença significativa $(p<0,05)$ nas contagens de L. monocytogenes entre estes dois tratamentos no $14^{\circ}$ dia de armazenamento, demonstrando que a nisina e o Biomax $\mathrm{D}^{\circledR}$ apresentaram o mesmo comportamento.

Avaliando-se a ação do Biomax $\mathrm{D}^{\circledR}$ contra $10^{5}$ UFC.g-1 de L. monocytogenes (Tratamento 6), verifica-se que a contagem celular, que foi de 4,24 log UFC.g-1 no tempo zero, passou a 3,74 log UFC.g-1 no sexto dia e 3,08 log UFC. $\mathrm{g}^{-1}$ no $14^{\circ}$ dia de armazenamento a $8^{\circ} \mathrm{C}$ (Quadro 1). Já no tratamento 5 (nisina contra $10^{5} \mathrm{UFC} . \mathrm{g}^{-1}$ de L. monocytogenes), a contagem de $L$. monocytogenes, que no tempo zero foi de 4,23 log UFC. $g^{-1}$, passou a 4,06 log UFC. $g^{-1}$ no sexto dia e a 3,19 log UFC. ${ }^{-1}$ no $14^{\circ}$ de armazenamento a $8^{\circ} \mathrm{C}$ (Quadro 1). Este resultado poderia sugerir que o Biomax $\mathrm{D}^{\circledR}$ (Tratamento 6) é mais eficaz contra L. monocytogenes do que a nisina (Tratamento 5) no sexto dia de armazenamento, no entanto, não 
houve diferença significativa $(p<0,05)$ nas contagens do micro-organismo entre os Tratamentos 5 e 6, demonstrando que ambos apresentaram eficácia semelhante. Ao se comparar a atividade dos antimicrobianos nisina e Biomax $\mathrm{D}^{\circledR}$ contra uma mesma concentração de L. monocytogenes em nata (Tratamentos 2 e 3 e Tratamentos 5 e 6), observa-se que as contagens do micro-organismo não apresentaram diferença significativa entre si $(\mathrm{p}<0,05)$ nos tempos zero, $6^{\circ}, 14^{\circ}$ e $20^{\circ}$ dia de armazenamento. Estes resultados demonstram que a nisina e o Biomax $\mathrm{D}^{\circledR}$, nas concentrações de 2,5 ppm e 1,5\%, respectivamente, apresentaram desempenho semelhante contra L. monocytogenes, tanto na concentração inicial de $10^{2}$, quanto de $10^{5} \mathrm{UFC} \cdot \mathrm{g}^{-1}$.

A atividade antimicrobiana do extrato de pomelo também foi relatada por outros autores. Gerhardt et al. (2012) avaliaram diversos extratos de frutas cítricas, inclusive extrato de pomelo, e verificaram atividade antimicrobiana destes extratos contra as bactérias Enterococcus faecalis, Escherichia coli, Salmonella Enteritidis, S. aureus e Pseudomonas aeruginosa.
Entretanto, nenhum estudo científico no Brasil avaliou o efeito do Biomax $\mathrm{D}^{\circledR}$ como antimicrobiano em alimentos em comparação à nisina. Neste estudo observou-se que o Biomax $\mathrm{D}^{\circledR}$ foi eficaz no controle de L. monocytogenes em nata, apresentando desempenho similar ao da nisina, um antimicrobiano já amplamente utilizado pela indústria láctea, portanto, apresenta potencial de utilização como antimicrobiano natural para uso em alimentos gordurosos.

\section{CONCLUSÃO}

A nata é um derivado lácteo com elevado teor de gordura, entretanto, L. monocytogenes pode se multiplicar e atingir elevadas contagens celulares, mesmo quando apresenta baixas concentrações iniciais. $\mathrm{O}$ Biomax $\mathrm{D}^{\circledR}$ possui atividade antilisteria semelhante à da nisina neste derivado lácteo, demonstrando potencial de utilização como antimicrobiano natural em alimentos gordurosos, como a nata.

\section{REFERÊNCIAS BIBLIOGRÁFICAS}

Aasen, I.M.; Markussen, S.; Moretro, T.; Katla. T.; Axelsson, L. e Naterstand, K. (2003) - Interactions of the bacteriocins, sakacin P and nisin with food constituents. International Journal of Food Microbiology, vol. 87, n.1-2, p. 35-43. http://dx.doi.org/10.1016/S0168-1605(03)00047-3

Bhatti, M.; Veeramachaneni, A. e Shelef, L.A. (2004) - Factors affecting the antilisterial effects of nisin in milk. International Journal of Food Microbiology, vol. 97, n. 2, p. 215-219.

http://dx.doi.org/10.1016/j.ijfoodmicro.2004.06.010

Brasil (2000). ANVISA - Agência Nacional de Vigilância Sanitária. Resolução no 23, de 15 de março de 2000 ementa: Dispõe sobre O Manual de Procedimentos Básicos para Registro e Dispensa da Obrigatoriedade de Registro de Produtos Pertinentes à Área de Alimentos publicação.Diário Oficial da União,16/03/2000. Anexo 1 e 2, p.23.

Brasil (2003). Ministério da Agricultura Pecuária e Abastecimento. Secretaria de Defesa Agropecuária. Instrução Normativa № 62, de 26 de agosto de 2003. Métodos analíticos oficiais para análises microbiológicas para controle de produtos de origem animal e água. Diário Oficial da União, 18 /09/2003, Seção 1, p. 14.

Brasil (2012). Ministério da Agricultura Pecuária e Abastecimento. Secretária de Defesa Agropecuária. Instrução Normativa no 23, de 30 de Agosto de 2012. Regulamento técnico de Identidade e Qualidade de nata. Diário Oficial da União, 31/08/2012. Seção1 p. 4.

Busatta, C.; Vidal, R.S.; Popiolski A.S.; Mossi, A.J.; Dariva, C.; Rodrigues, M.R.A.; Corazza, F.C.; Corazza, M.L.; Vladimir Oliveira, J. e Cansian, R.L. (2008) - Application of Origanum majorana L. essential oil as an antimicrobial agent in sausage. Food Microbiology, vol. 25, n. 1, p. 207-211. http://dx.doi.org/10.1016/j.fm.2007.07.003

Cossart, P. (2011) - Illuminating the landscape of host-pathogen interactions with the bacterium Listeria monocytogenes. Proceeding of the National Academy of Sciences of the United States of America. vol. 108, n. 49, p. 19484-19491. http://dx.doi.org/10.1073/pnas.1112371108

Gerhardt, C.; Wiest, J.M; Girolometto, G.; Magnólia, M.A.A.S. e Weschenfelder, S. (2012) - Aproveitamento da casca de citros na perspectiva de alimentos: prospecção da atividade antibacteriana. Brazilian Journal of Food Technology, vol. 15, p. 11-17. 
Jack, R.W.; Tagg, J.R. e Ray, B. (1995) - Bacteriocins of gram-positive bacteria. Microbiology Reviews, vol. 59, n. 2, p. 171-200.

Pimentel-Filho, N.J.; Mantovani, H.C.; Carvalho, A.F.; Dias, R.S. e Vanetti, M.C.D. (2014) - Efficacy of bovicin HC5 and nisin combination against Listeria monocytogenes and Staphylococcus aureus in fresh cheese. International Journal of Food Science \& Technology. vol. 49, n. 2, p. 416-422. http://dx.doi.org/10.1111/ijfs.12316

Prozyn (2013) - Biomax D: Substituição Natural aos Conservantes; Guia do fabricante.

Tenenhaus-Aziza, F.; Daudin, J.J.; Maffre, A., e Sanaa, M. (2014) - Risk-based approach for microbiological food safety management in the dairy: The case of Listeria monocytogenes in soft cheese made from pasteurized milk. Risk Analysis, vol. 34, n. 1, p. 56-74. http://dx.doi.org/10.1111/risa.12074 